\title{
Insertion of Vagus Nerve Stimulation in a Child with Left Sided VP Shunt
}

Mahmoud Taha ${ }^{1^{*}}$ and Raida Albaradi ${ }^{2}$

${ }^{1}$ Department of Neurosurgery, King Fahad Specialist Hospital-Dammam, Saudi Arabia

2Department of Pediatric Neurology, King Fahad Specialist Hospital-Dammam, Saudi Arabia

"Corresponding author: Mahmoud Taha, Consultant Neurosurgeon, Department of Neurosurgery, King Fahad Specialist Hospital-Dammam, Amer Bin Thabet St. Dammam 31444, Kingdom of Saudi Arabia, Tel: 001966 38431111; Fax: 001966 38423641; E-mail: mtaha66@hotmail.com

Received date: Jul 18, 2014, Accepted date: Sep 06, 2014, Published date: Sep 15, 2014

Copyright: ( 2014 Taha MG, et al. This is an open-access article distributed under the terms of the Creative Commons Attribution License, which permits unrestricted use, distribution, and reproduction in any medium, provided the original author and source are credited.

\section{Abstract}

Background: Vagus nerve stimulation is an adjunct device used for the treatment of drug-resistant (refractory) epilepsy. Insertion of this device is usually straightforward in patients with no previous surgery or scar in the left side of the neck or chest wall.

Methods: We present a case of 6-year-old girl who had refractory seizures and left-sided programmable ventriculo-peritoneal shunt. VNS device were inserted and several modifications to the standard surgical procedure were made in order to avoid the distal catheter of the shunt in the neck and in the anterior chest wall.

Results: The child had no immediate complication related to her VP shunt or her VNS device at discharge or at 3 and 6 months follow up. The stimulation was successfully initiated 2 weeks post operatively. Her seizure control improved slightly at 6 months of stimulation.

Conclusions: The technical modification that we have performed is safe and useful in patients undergoing insertion of VNS with left sided shunt or other implant in the left side of the neck or chest wall.

Keywords: Vagus nerve stimulation; Refractory epilepsy; Left VP shunt; Programmable VP shunt

\section{Introduction}

Vagus nerve stimulation (VNS) was approved in Europe in 1994 for treating refractory epilepsy in patients over the age of 12 and in 1997 by the US Food and Drug Administration (FDA). However, early studies by Murphy et al. [1] suggested that VNS was well-tolerated by children under the age of 12 and could result in a dramatic reduction in the seizure burden [1]. The efficacy appears to improve if the system is used for a longer period [2].

Although the mechanism of action of VNS is still not fully understood, it was felt that continual stimulation of the vagus nerve by an implantable electrical device might result in a widespread bilateral disruption of the hyper-synchronous brain electrical activity that caused seizures. The reason for this lies in the neuro-anatomical connections of the vagus nerve as afferent fibres from the vagus nerve which comprise about $80 \%$ of the axons in the cervical vagus nerve, terminate in the nuclei of the ipsilateral medulla and in the contralateral nucleus of the tractus solitaries. Inputs to these nuclei are then conveyed to widespread bilateral areas of the cerebral cortex, diencephalon and limbic system. The effects of VNS on these brain areas have been confirmed by positron emission tomography and functional MRI studies [3].

The implantation procedure is straightforward. The first step involves creation of the chest pocket that accommodates the pulse generator usually in the left side of the chest wall below the clavicle or more laterally at the anterior axillary line. Next, through a separate incision in the left side of the neck at C5-6 level, the carotid sheath is opened, and the vagus nerve trunk isolated. The lead is tunnelled within the subcutaneous tract between the two incisions. The helical electrodes are applied to the nerve and the lead connector pins are secured to the generator. After additional electro-diagnostic testing, the lead and generator are secured to adjacent tissue, and the wound is closed in standard multilayer fashion [4].

The clinical VNS device allows a noninvasive magnet held near the pulse generator to program current intensity, individual pulse duration, pulse frequency, on-off cycle time and intensity and duration of any extra pulse.

Pulse width of 0.25 ms may be better tolerated than those of 0.5 , with similar efficacy, but $0.13 \mathrm{~ms}$ pulses are less effective. Stimulation at frequencies below 20 per second may allow increased stimulation of un-myelinated C-fibers, with more autonomic side effects [5].

\section{Case report}

A 6-year-old girl with medically resistant epilepsy was referred to our department for consideration of VNS insertion. She is known case of Epidermal Nevus Syndrome and refractory seizures. Her seizure onset is at 3 years of age. She has two types of seizures in the form of right sided focal seizures and generalized tonic clonic seizures occurring once every 1-2 weeks. The child is a product of full-term, normal spontaneous vaginal delivery, birth weight was $3 \mathrm{~kg}$. At the age of 4 months the child had ventricular-peritoneal shunt inserted for congenital hydrocephalus.

She was treated with steroid for the epidermal nevus at the age of one year. Her developmental milestone is delayed. There is no family history of similar illness. Her seizures were treated with several 
antiepileptic drugs which include Valproic acid, Carbamazepine, and Levetiracetam without any significant improvement.

The shunt was revised several times for repeated blockages and her last one was a programmable type inserted in the left side.

Her physical examination revealed active and pleasant little girl with no dysmorphic feature. She has resolving left facial epidermal nevus in the left side of the face. Her systemic examinations are normal. The neurological examination revealed mild right sided hemi-pareses.

MR imaging of the brain showed severe left hemispheric cortical dysplasia (Figure 1).

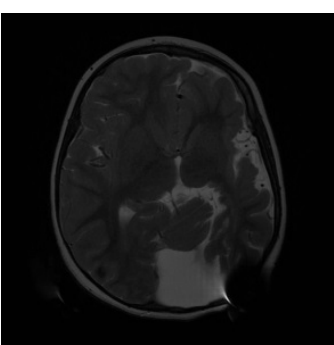

Figure 1: MRI brain of the patient showing severe left hemispheric cortical dysplasia.

NM/PET CT 18F-FDG Brain Scan revealed diffuse decrease FDG metabolic rate involving the left hemisphere. The interictal SPECT showed hypoperfusion over the same region (Figure 2). Her video electroencephalogram revealed frequent epileptic discharges over the left frontal, temporal and parietal regions and slowing over the left hemisphere. She has several typical seizures arising from the left frontal, temporal, and parietal areas.

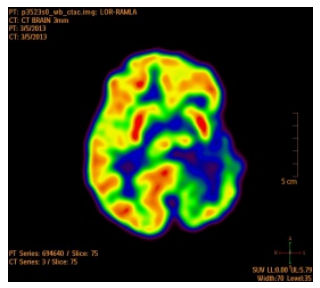

Figure 2: NM/PET CT 18F-FDG Brain Scan revealed diffuse decrease FDG metabolic rate involving the left hemisphere

The case was discussed in our epilepsy surgery meeting and several options were considered. However, after consulting the family they agreed on VNS insertion.

\section{Operative Technique}

The procedure was done under general anaesthesia and we used the Demipulse generator (Model 103) Cypronics.

Certain technical modifications were made in this case:

The left neck incision was made below the usual site at C6-7 vertebral level to avoid the contact with the shunting tube.
The pulse generator was inserted in the right side of the chest wall to avoid injuring the shunt or the generator in case of future shunt revision.

The tunnelling was made in a curved fashion to avoid crossing the trachea or the supra-sternal notch and causing future discomfort (Figures 3 and 4).

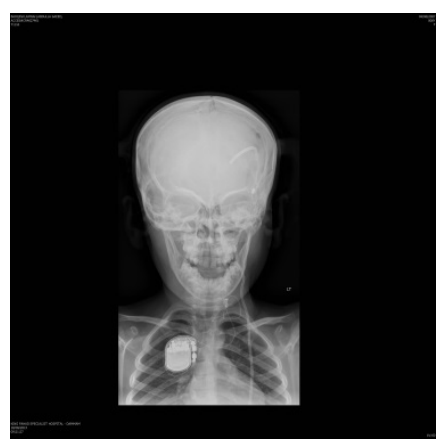

Figure 3: Plain X-ray after insertion of the VNS showing the left VP shunt and the right-sided generator of the VNS devise.

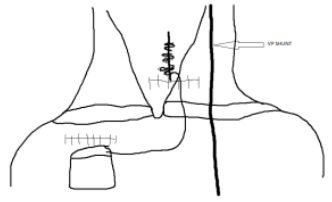

Figure 4: Diagram of the surgical modification

The patient did well after surgery with no problems regarding her shunt or her VNS devises were detected 6 months after surgery. Her seizure control improved slightly at 6 months of stimulation in terms of frequency and intensity.

\section{Discussion}

The presence of left side VP shunt in patients undergoing VNS insertion can make the procedure more challenging for several reasons:

The presence of the distal tube of the shunt can make the dissection of the carotid sheath more difficult. This is the reason why we advise that the incision is more inferior to the standard level (at C5-6 level) in order to avoid the scaring around the shunt tube and to avoid injuring the tube itself.

Placing the pulse generator in the left side of the chest wall can cause damage to the distal tube of the shunt and can theoretically create more problems when revising the shunt or the generator in the future. Therefore placing the generator in the right side would be much safer.

By placing the generator in the right side of the chest wall, the electrode has to be passed from left to right across the midline. We advise that the tunnelling device should be curved and passed below the sternal notch to avoid placing the leads in front of the trachea which might cause some discomfort to the patient postoperatively. 
Citation: Taha M and Albaradi R (2014) Insertion of Vagus Nerve Stimulation in a Child with Left Sided VP Shunt. J Neurol Neurophysiol 5: 229. doi:10.4172/2155-9562.1000229

Page 3 of 3

The alternative approach is to insert a right-sided VNS. A smal number of studies showed some good results but using the right vagus nerve for seizure control is not well established [4].

\section{Conclusion}

This modification to the standard technique of VNS insertion was not published previously, and we feel that it is safe and useful in patients with left sided shunts or other implants.

\section{References}

1. Murphy JV, Hornig G, Schallert G (1995) Left vagal nerve stimulation in children with refractory epilepsy. Preliminary observations. See comment in PubMed Commons below Arch Neurol 52: 886-889.
2. Crumrine PK (2000) Vagal nerve stimulation in children. See comment in PubMed Commons below SeminPediatrNeurol 7: 216-223.

3. Guberman A (2004) Vagus nerve stimulation in the treatment of epilepsy. See comment in PubMed Commons below CMAJ 171: 1165-1166.

4. Amar AP, Levy ML, Apuzzo MLJ (2004) Vagus Nerve Stimulation for Intractable Epilepsy, in H. Richard Winn, YOUMANS - Neurological Surgery, 5ed. Philadelphia: WB Saunders 2: 2643-2650.

5. Fisher RS (2012) Therapeutic devices for epilepsy. See comment in PubMed Commons below Ann Neurol 71: 157-168.

6. McGregor A, Wheless J, Baumgartner J, Bettis D (2005) Right-sided vagus nerve stimulation as a treatment for refractory epilepsy in humans. See comment in PubMed Commons below Epilepsia 46: 91-96. 